\title{
Design and Implementation of Plan Management Based on Manufacturing Execution System
}

\author{
Liu Sha ${ }^{1,}$ a , Li Peng ${ }^{1}$, Hu Guolinag ${ }^{1}$, Duan Bin ${ }^{1}$ \\ ${ }^{1}$ Department of Electrical Engineering and Automation, Luoyang Institute of Science and \\ Technology, Luoyang, 471023, China \\ aemail: liusha@sia.cn
}

Keywords: MES; plan management; production management; scheduling

\begin{abstract}
This essay is aiming at the field of specific applications for aluminum electrolysis, building up a manufacture planning management system based on a kind of factory module as well as MES system platform and the process of achieving plan management. The plan management model covering the entire project life cycle, including planning, implementation plans, and the implementation of the plan after feedback on the implementation of the development plan which includes the development of plans for the development and planning information indicators. This model has a certain universality and ease of use, but also for other industries program management.
\end{abstract}

\section{Introduction}

Along by the development of scientific technology, the combination between traditional industries and the computing operations, aluminum electrolysis which get a great influence from it, especially the operation process of its manufacturing skills, from the very beginning of cell voltage control to the cell resistance, and timing-adding electrolysis of aluminum oxide until recent years focus on the balance electrolysis batch and energy balance, meanwhile to constantly give out the optimized design of cell voltage[1], which ,enhance the manufacturing of aluminum electrolysis.

Our country's development state of aluminum electrolysis can be divided in to following statements [2]: Using computer to make some simple movement; Adaptive control stage; Develope an expert system and the implementation stage.

The key to realize enterprise resource optimization is to establish the internal supply chain including production, supply and sales of the entire enterprise, which has the enterprise management system optimization function [3].

The enterprise production planning is about the general aspects of the plan of production system. Plan has an important position for the production; enterprises in other executive management are closely around the planning and deployment. Production planning refers to the production plan of the concrete plan, it will be determined by [4] a series of comprehensive balance work to finish production plan. Perfect production plan work, for the enterprise is production of security.

\section{Plan Management}

Due to the limitation of the tools and methods, the complexity of enterprise system, it is a gradual process of understanding enterprise resource optimization, way to realize enterprise resource optimization and thus also presents diversity. In CIMS and ERP support, the final completion of the optimization target [3] enterprise resource limited.

There are three core programs, respectively is the master production schedule (MPS), material requirements planning (MRP) and capacity requirements planning (CRP).

The main production plan, from a management point of view definition is the enterprise is in a period of time on specific products or components of the production plan, the plan object is directly external sales of independent demand item or assembly; from the perspective of system design is defined is the enterprise order and forecast demand for reasonable optimization arrangement in the specified within the specified time, production time and quantity of each product. The main 
production plan set can use the formula 1 represents [5]:

$M P S=\{(p, q, b d, e d, b b, e b)\}$

Among them, $\mathrm{P}$ is the planned product, $\mathrm{Q}$ product plan number, BD for the program start date, ed for the scheduled completion date, BB plans to start time, EB for the scheduled completion time. MPSi said the article I of the main production plan, as shown in equation 4.

$M P S_{i}=\left\{(p, q, b d, e b, b b, e b)_{i}\right\}, M P S_{i} \in M P S$

The definition of material requirements planning as shown in equation 3.

$M R P=\{(p, q, b d, e d, b b, e b, p f)\}$

Among them, $\mathrm{P}$ is the program for parts, Q products plan number, BD plans to start date, ed for the scheduled completion date, BB plans to start time, EB for the scheduled completion time, PF said the plan state. MRPi said the article I of material requirement planning, as shown in equation 4.

$M R P_{i}=\{(p, q, b d, e d, b b, e b, p f)\}, M R P_{i} \in M R P$

The plans establish goals and objectives for the organization, and find the best way to achieve. According to ERP system sales orders, sales forecast, finished goods inventory, in advance of other kinds of basic information, select the appropriate parameters, calculate the production plan feasible corresponding period; the calculation results of MPS, passed to the MRP and other related programs as basic data, through the BOM (product BOM) expansion, introducing the work center information to determine the real demand of raw materials and process information, combined with the inventory information for purchasing plan and job shop scheduling. The entire supplements chain to plan as the core, through the planning system to the supply chain series to achieve the integration of production and marketing of [6].

In general, the production plan management process mainly includes five steps[7]:

- Preparation: Including accounting demand forecasting and production capability of the enterprise. Demand forecasting and production capacity of accounting, is the main basis for production planning.

- Plan Index: Refers to the planned period shall complete product variety, quality, yield and output value targets of the plan.

- Check and balance Production Capacity: The first part of the work to get information, make specific production capacity of accounting, and the balance of various factors, to get the best production plan.

- Determine the Product Production Schedule

- Implementation of the Organization and Check the Production Plan: The implementation of the organization and check the production plan

Production plan according to the length of plan period, it can be divided into long, medium and short term plan:

-Long-Term Plan: Also known as the strategic planning, responsible for corporate senior leadership, focus on the development of enterprises and the external environment, this level involves many uncertain factors are summarized, the index can only be;

- Medium-Term Plan: By the competent department is responsible for the production, the purpose is to effectively use the existing resources, which also contains many uncertain factors;

- Short-Term Plan: Determined according to the production task, capacity and material supply specify the details of the plan, in order to ensure the realization of upper [8] plan.

Production planning and control of production determines the limited resource acquisition, use and distribution, in the most effective way to meet the needs of the customers of [9].

\section{Scheme Design}

Consider the special nature of aluminum electrolysis, in the production of aluminum electrolysis, electrolysis groove state influence the liquid aluminium production directly, not fully human intervention, so make a perfect plan, and the plan management in combination with other function becomes particularly important. Given a combination of planning index management, scheduling 
management, scheduling management, implementation of planning management daily operation management, in the electrolytic aluminum production is very necessary and feasible [9].

This part is applied to manufacturing execution system platform design plan management, plant architecture model is the premise of the design, the manufacturing execution system constructed.

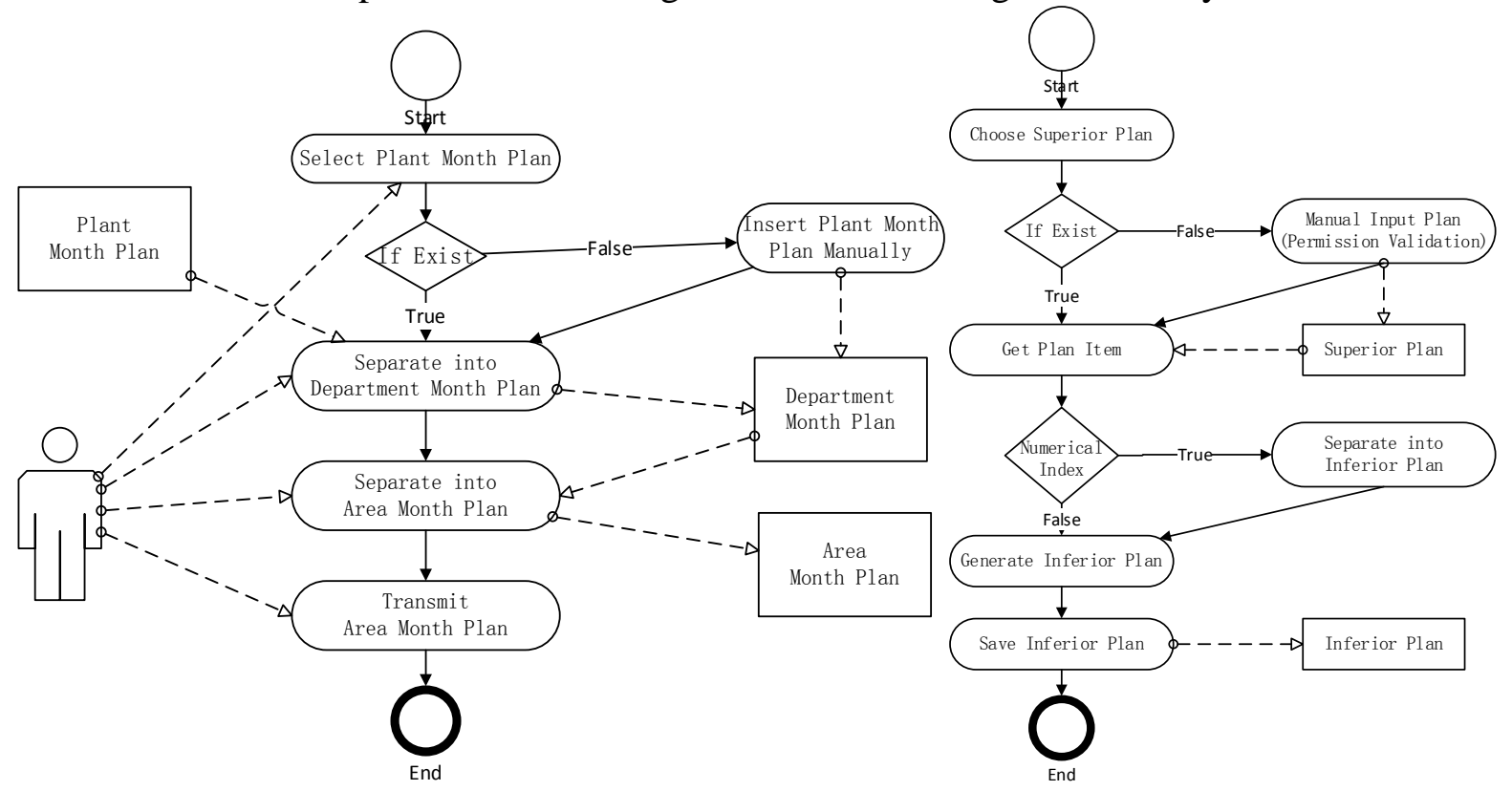

Fig.1. Plan Decomposition Flow Chart

\section{A. The Overall Model Design}

The model design of the entire planning process for the decomposition of from top to bottom, step by step, from the first step feedback.

For a specific plan period, initial plans for the supervisory plan, develop level plans to guide the workshop plan, make workshop planning guidance work area planning area, planning guidance area mission planning task, work area to guide the practical production; after task execution, layer by layer upward feedback information until the top floor plan, namely the supervisory plan, the actual completion of planning area the amount of feedback to the task information, and task status changed to "completed", then the mission completed amount of feedback to the area plans to complete the final amount, information feedback to the workshop plans to complete the amount and quantity plan finished factory.

\section{B. Process Design}

According to the above plan contains the elements of information, in view of the aluminum electrolysis project, the commencement date, date of completion, start time, completion time information can be included in the planned date.

In order to realize the flexible planning system, according to a plan view of the plan, the layer decomposition method, here illustrated by means of specific monthly plan. Decomposition methods, where each layer is divided into two kinds, can according to the number of existing subordinate departments, electrolytic tank electrolytic tank current situation and other information, can also be free to modify the. After the automatic decomposition of superior plan, through the command, these plans issued to the next level departments.

The monthly plan is divided into three levels, plant, workshop areas' plan and working area plan. From the beginning of plant monthly plan, if there is no plant monthly plan, here can through the plant level plan personnel manually add and issued; decomposition of plant has been issued monthly plan to the workshop, the workshop plan generation and preservation, and then through the command passed to subordinate planners; similarly, the lower program staff received plant issued by workshop planning, the decomposition and save the working area plan, and then assigned to the work area.

Area of workshop area received the formulation of plans, formulate and comprehensive planning information and its electrolytic groove guide the daily task scheduling. 
Split each plan contains automatic splitting and manually split the two forms.

Main production plan index, yield, quality, variety of output and production period of [8], including such as aluminum quantity yield index, its plan for counting type plan, plan amount lower all plans should be the sum of the superior plan amount, so use in automatic split weight splitting method.

Weight splitting method, namely according to the electrolytic tank situation information of each different subordinate, give a plan to split index Xi I, then by all sectors of the index are standardized, get a set of coefficients of $[0,1]$, namely bi. To determine the split weight gamma I through the coefficient, in this process, can make artificial adjustment, but the need to standardize again. According to the known weights of allocation plan for different amount of subordinate departments, standardization as shown in equation 5.

$$
b_{i}=\frac{1}{\sum\left\|\xi_{i}\right\|} \xi_{i}
$$

While such as electrolytic temperature and other non production index, automatic resolution method of the plan as a guide method, that is, without making any change, consistent with the higher departments plan.

$$
\mathrm{N}\left(p_{i}\right)=\left\{\begin{array}{cc}
\gamma_{i} N(P), & P \text { is Numerical Item } \\
N(P), & P \text { is not Numrical Item }
\end{array}\right.
$$

As shown in equation 6 , according to the plan of information a yield index, as a single superior planning information, namely the information of a single plan items a specific plan period of a department, such as the 2014 July second electrolysis plant aluminum tapping volume plan; to set lower plan higher level planning information, similar to the superior plan information. As of 2014 July second electrolytic factory subordinate all workshop sets of aluminum content plan; as the number of lower level planning information value, can see intuitively, the superior department plans a number lower resolution Department oriented, i.e. second electrolytic plant, workshop a number that contains a plan index plan; for the higher level planning information the term junior program information, namely the second electrolysis factory, a workshop of the plan targets planning information.

For the split weight plan, used to guide the superior plan to split the lower plan, and. From the set point of view, the logic relation plan as shown in equation 7 , for the same plan period, each set of subordinate plans a plan all belong to the whole lower plan, set the lower planning approaches or equals to the superior plan.

$$
p_{i} \in p, p \rightarrow P
$$

The amount of information for resolution plan, plan metrics program for numerical information. In the planned amount of each add and modify, save at the same time, the amount of the system to automatically modify the plan to split the superior plan, to ensure the real-time and accuracy higher resolution volume plan.

After the program automatically split, editors allow in the task before, according to the needs to manually adjust the amount of plan. According to the plan information yield index, completely decomposed to guarantee it can increase the amount, split the elements in the model, used to real-time preservation and display the current plan information has split the sum of the number, as shown in equation 8:

$$
\mathrm{S}(\mathrm{P})=\sum_{i=0}^{n} N\left(p_{i}\right)
$$

Here the amount increased resolution plan, split works can guide the superior plan, not only to ensure that the complete superior plans to split, namely all planned decomposition, also can by viewing the removed component, obtain plans to split the information quickly and intuitively, and have split the superior plan modifications to provide convenient.

\section{Planning Design}

In order to ensure the plan information robustness and versatility, and combining the particularity of the aluminum electrolysis industry, design the following plan information structure.

The overall design plan information can be related to the following five categories, plan item, plan item type, unit information, plan information, plan item data. The plan information related to the plan item data, plan item data association to plan item, plan item related to the plan item types 
and units, the design of such a class implements plan information storage form of flexible, increases the versatility of the plan management model.

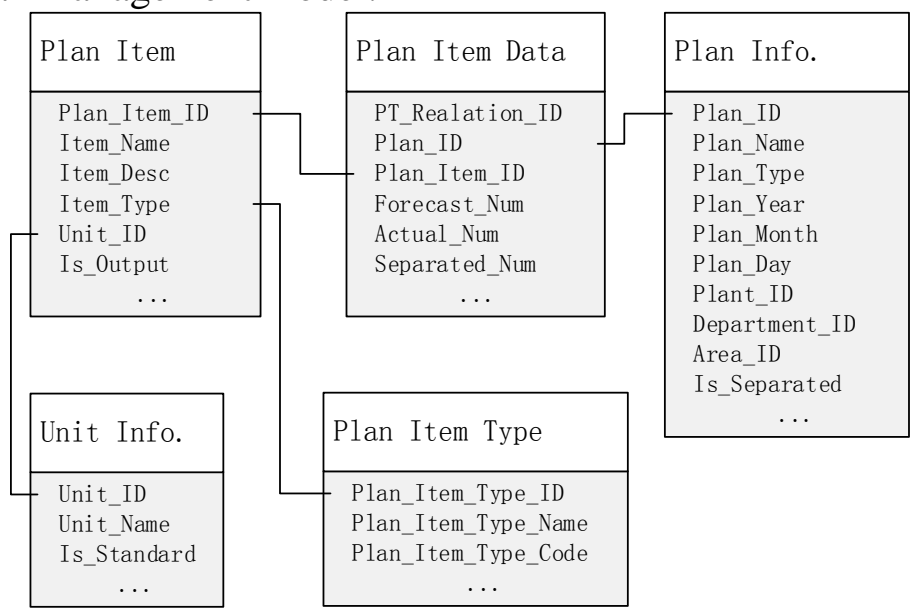

Fig.2. Class Diagram

\section{Modules}

A. Program Instructions of Process

If the plan system does not contain has issued the plant planning, then planning process from step (1) to start, if it contains has issued the plant planning, then planning process from step (2) to start.

1) Part of plant planning:

a) According to the demand of plan index, including the selected indexes;

b) According to the situation, equipment status, electrolyzer plant personnel, and feedback the plan period production, factory to obtain overall production capacity;

c) According to the ability of overall production factories, with external demand, according to the plan targets set, making plans for the plant;

d) Plan is completed, plans.

2) Levels of subordinates department plans to part

a) Gets higher planning information;

b) According to the Department of different subordinate department of personnel and equipment, to obtain lower production capacity of information;

c) According to the subordinate department of production capacity, the superior plan decomposition;

d) Plan is completed, plans.

B. The Formulation of the Plan Index

This system made the plan index with the addition of editing, policymakers must fill includes plan targets, plan index type and other information, to ensure the perfect plan index, and used to develop the guidance plan.

C. Program Decomposition of Sample

Level resolution monthly plan, from the beginning of the year and month selected factories. If not the existence of the monthly plant monthly plan, use "add" function to manually add plant monthly plan, add after the automatic decomposition of the plant monthly plan will lower the data table, decomposition follows the method mentioned in the third chapter of the step.

If the existing plant monthly plan, and no decomposition, then choose the specified factory, year and month, the upper part of the table of data display plant monthly plan information, lower data table display automatic decomposition of information.

If the existing plant monthly plan, and has been decomposed, namely the presence of decomposition workshop monthly plan information, then the selected factories, year and month, the upper part of plant level data table shows the monthly plan information, lower data table shows have been decomposed shop monthly plan information. 
After the workshop monthly plan after modification has been shown, save the information and release these workshops monthly plan, namely the decomposition of plant level monthly plan to shop monthly plan completed.

\section{Conclusion}

According to the general and special aluminum electrolysis industry, constructs a plan to split with automatic and Manual issued model, this model provides superior plan formulation of the underlying program split, and contains different types of plan index plan automatically split and manually split, this model can also provide information to split regulation function, to ensure perfect plan issued by the information decomposition.

\section{Acknowledgement}

This work is supported National High-tech R\&D Program of China (863 Program) “Aluminum Electrolytic MES Research and Application Based on the Accurate Perception and Intelligent Decisions” (2013AA040705).

\section{References}

[1] K. Grjotheim and H. Kvande, Introduction to Aluminum Electrolysis--Understanding the Hall-He',roult Process, 1993 :Aluminum-Verlag.

[2] Cao D, Yang B, Li J, et al. Research on decision support system of aluminum electrolysis process based on data warehouse[C]. Control and Decision Conference (CCDC), 2010 Chinese. IEEE, 2010: 3828-3831.

[3] Severo D S, Schneider A F, Pinto E C V, et al. Modeling magnetohydrodynamics of aluminum electrolysis cells with ANSYS and CFX[J]. Light Metals, 2005, 475: 480.

[4] $\mathrm{Hu}$ Y, Zhang D S, Liu F. Study on the optimization method of enterprise resources with fuzzy constraint variables[C]. 2009 International Conference on Machine Learning and Cybernetics. 2009, 5: 2702-2707.

[5] Magee J F, Boodman D M. Production planning and inventory control[J]. 1967.

[6] Khalil T, Dominic P D D, Fadzil bin Hassan M. Decision support system framework for implementation of Enterprise Resource Planning (ERP) system[C]. Information Technology (ITSim), 2010 International Symposium in. IEEE, 2010, 3: 1439-1443.

[7] Windt K, Jeken O. Autonomous product manufacturing cycle-an integrated approach to process planning and production control[C]. Industrial Engineering and Engineering Management (IEEM), 2010 IEEE International Conference on. IEEE, 2010: 1286-1290.

[8] Stephen C. Graves, Manufacturing Planning and Control[J]. Massachusetts Institute of Technology, 1999: 17-25.

[9] Younus $\mathrm{M}$, Peiyong $\mathrm{C}$, $\mathrm{Hu} \mathrm{L}$, et al. MES development and significant applications in manufacturing-A review[C]. Education Technology and Computer (ICETC), 2010 2nd International Conference on. IEEE, 2010, 5: V5-97-V5-101 